\title{
Rumor Retransmission on Twitter: Message Characteristics, user Characteristics and Retransmission Outcomes
}

Alton Chua, Xiaoyu Chen

Nanyang Technological University

Singapore

\{altonchua@ntu.edu.sg\}\{XIAOYU001@e.ntu.edu.sg\}

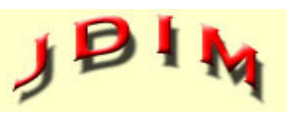

Journal of Digital Information Management

\begin{abstract}
This paper seeks to depict online rumor retransmission using three main constructs, namely, message characteristics, user characteristics and retransmission outcomes. In particular, it teases retransmission outcomes into volume, immediacy and lifespan of rumor tweets. The dataset was drawn from 322 original Twitter messages which generated some 5,700 retweets about the rumored death of Lee Kuan Yew (LKY). Content analysis and statistical tests were conducted. The results confirm the robustness of the research model. Specifically, rumor message characteristics affect retransmission in terms of volume, immediacy and lifespan. Additionally, user characteristics are positively related to volume and immediacy. Finally, user characteristics have a moderating effect on the relationships between rumor message characteristics and retransmission outcomes. This paper illustrates the applicability of the information diffusion model on online rumor retransmission which hitherto has yet to be attempted. Moreover, it granularizes the concept of online rumor retransmission into three important outcomes, namely, volume, immediacy and lifespan. On the practical front, this paper has implications for organizations seeking to combat rumors.
\end{abstract}

Subject Categories and Descriptors:

[H.3.5 Online Information Services]; Data sharing

General Terms: Social Media, Social Networks, Information Transmission, Text Analysis

Keywords: Rumor, Retransmission, Twitter, Information Diffusion

Received: 3 October 2019, Revised 29 December 2019, Accepted 31 December 2019
Review Metrics: Review Scale: 0-6, Review Score: 4.62, Interreviewer consistency: $89.5 \%$

DOI: $10.6025 / \mathrm{jdim} / 2019 / 18 / 1 / 21-32$

\section{Introduction}

Twitter, which boasts some 47 million monthly active users in the U.S. alone (Statista, 2019), is one of the most popular social media for sharing news (Honeycutt, et al., 2009, Kwak et al., 2010). This means that it can also be used to spread rumor. In fact, one of the effective features that enables rumors to be circulated quickly is retweeting. Previous research suggests that retweets could potentially reach an average of 1000 users in a short time (Kwak, et al. 2010). Since retransmission is an inherent part of rumor-mongering, understanding the factors that contribute to retransmission of online rumors might eventually shed light on possible control mechanisms.

Factors fueling online rumor retransmission can be categorized into rumor message characteristics and user characteristics. The former encompasses features such as the use of images, and expressions of emotions (Liu, et al., 2014; Oh, et al., 2013). A rumor with attached images may look compelling, and hence attract retransmission. Likewise, when couched in emotionally-laden phrases such as "scared" and "sad", a rumor can tug at users' heartstring and turns viral (Oh, et al., 2013). User characteristics include the user's connectivity and activity in a network (Chen, 2011). Conceivably, a rumor posted by an active Twitter user with a significant number of connections may have a higher probability of being retransmitted than one by someone new to the network.

Rumor retransmission is a form of information diffusion. 
Yet, except for a few works (Mozafari \& Hamzeh, 2015), there is a lack of research that stands at the intersection of rumor theory (Oh, et al., 2010) and information diffusion literature (Stieglitz \& Linh, 2013), while at the same time being attentive to the peculiar affordances of social media. For this reason, the intent of this paper is to examine online rumor retransmission primarily through the lens of information diffusion. Such a perspective is taken because the way rumors spread is comparable to how new ideas propagate in a social system (Lee et al., 2015) and how product review information flows via word-of-mouth (Kimmel, 2013). Additionally, in the context of Twitter use, two other theoretical perspectives, namely, the Elaboration Likelihood Model (Cacioppo \& Petty, 1984), and opinion leadership, are brought to bear.

Underpinned by these theories, this paper seeks to depict online rumor retransmission using three main constructs, namely, message characteristics, user characteristics and retransmission outcomes. In particular, it teases retransmission outcomes into volume, immediacy and lifespan of rumor tweets (Suh, et al., 2010). Volume is an indication of the interest generated by a message. Immediacy measures how quickly a message attracts attention. Lifespan marks the duration within which a message remains active in the network. As previous work has shown that user characteristics indirectly affect information diffusion on Twitter (van de Velde, et al., 2015), the moderating effect of user characteristics on message characteristics will also be explored. Thus, the three research questions are as follows:

RQ1: How do rumor message characteristics affect retransmission of rumor tweets in terms of volume, immediacy and lifespan?

RQ2: How do user characteristics affect retransmission of rumor tweets in terms of volume, immediacy and lifespan?

RQ3: How do user characteristics moderate the relationship between rumor message characteristics and retransmission of rumor tweets in terms of volume, immediacy and lifespan?

This paper is part of a larger research project which aims to elucidate the retransmission mechanisms of rumors on social media. In past studies, we have explored the role of source and content credibility (Chua, Tee, Pang, \& Lim, 2016) and the correction messages (Chua, Tee, Pang, \& Lim, 2017). However, the current paper extends previous works in two ways. First, this paper integrates the effects of message characteristics and user characteristics on the retransmission outcomes. Second, to delve deeper, we conceptualize the retransmission outcomes in terms of three dimensions, namely, volume, immediacy and lifespan. As with previous studies, the dataset was drawn from the case of the rumored death of Lee Kuan Yew (LKY), a widely-regarded Singaporean political figure prior to his actual demise. This case was chosen because the rumor content spread quickly on Twitter and attracted local and international attention. In general, rumors are messages whose veracity could not be confirmed at their point of circulation.

This paper makes two theoretical contributions. First, it illustrates the applicability of the information diffusion model, along with ELM and opinion leadership on online rumor retransmission. Such an undertaking has yet to be attempted hitherto. In particular, it shows that user characteristics exert a direct as well as indirect (moderating) effect on retransmission. Next, it granularizes the concept of online rumor retransmission into three outcomes, namely, volume, immediacy and lifespan. These are related but distinct, and have so far been neglected in previous studies. Together, they paint a fuller picture of the fallout, and hold implications for combating online rumors.

The remainder of this paper is organized as follows: The next section provides an overview of rumor research, information diffusion literature and the theoretical underpinnings of rumor retransmission. It culminates in a proposed rumor retransmission model which comprises three main constructs, namely, rumor message, user and retransmission. The Methodology section explains the procedure for data collection, operationalization and analysis. The results are presented next. This is followed by a discussion of the results. Finally, the paper concludes by highlighting its implications and limitations.

\section{Literature Review}

\subsection{Rumor Research}

Rumors are commonly conceived as unverified information which arises during uncertainties. Some people spread rumors to make sense of the situation (DiFonzo \& Bordia, 2017), while others do so to serve their self-interests, boost their status or just to wreak havoc (Marett \& Joshi, 2009). In the social media era, the spread of rumor is greatly enhanced. This is largely due to the low cost of content production and the lean information carried on social media that makes it hard for users to assess the veracity of the content.

Twitter, a popular micro-blogging platform, has been an important data source for most research rumor. This is no surprise as Twitter carries unique affordances that make it an ideal conduit for quick information dissemination to a large audience. Social conformity influences Twitter use especially in the wake of a rumor, or an outbreak of a crisis. Tweets can be spontaneously created and instantly shared.

A common theme in rumor research is why and how rumor spread (Zeng, et al., 2016). The foci are on factors that fuel rumor-mongering which include anxiety, personal involvement and message properties (Oh, et al., 2013; Lee, et al., 2015; Pezzo \& Beckstead, 2006). Additionally, 
the dynamics of rumor spread has been studied using measures like burst patterns and time-series progression (Maddock, et al., 2015), as well as models such as Spreaders-Ignorants-Stiflers (SIR) (Zhang \& Chen, 2014).

Another related theme is the users' response to rumor. For example, using a multi-features model, users' views during a rumor-outbreak could be classified in terms of agreement, disagreement, comment and query (Ma \& Luo, 2019). Similarly, another study compares expressions of credence and emotions among rumor and counter-rumor messages (Pal et al., 2017).

In contrast, research that investigates rumor-mongering through retweets is less common. The retweet function of Twitter offers a simple yet highly effective way for a rumor to spread. As easy as clicking a button, retweeting demands little physical and cognitive effort on a user's part. And for highly connected users, the promulgation can be far and wide. This is why studying rumor retransmission through retweets is particularly significant.

\subsection{Information Diffusion Literature}

Given that rumor retransmission is a form of information diffusion, a review of literature on information diffusion helps clarify what rumor retransmission entails. The flow of information through a network is similar to the spread of disease within a community. Epidemiology and information diffusion, therefore, share a common thread. Most disease-propagation models are based on the cycle of disease (Bailey, 1975). First, a person is susceptible. Next, when exposed to the disease, the person may be infected. This person could in turn become a carrier and spread the disease to others. Finally, the person recovers. In the context of information diffusion, the disease is the message, and the one infected is the one who receives the message. A carrier is one who passes the message to the next person.

Another way to study the flow of information has been done through a game-theoretic approach. Modelling after the game of coordination, individuals' choices between two options ( 0 and 1 ) can be shown mathematically to be affected by the decision of their neighbors (Morris, 2000). Contagion occurs most likely if there is low neighbor's growth and that local interaction is sufficiently uniform. In other words, when applied to information diffusion, the premise is that users will enjoy an increase in utility by accepting new information if a sufficient number of their friends also do likewise.

The spread of information can also be viewed as the propagation of an innovation through a social network. From a sociological perspective, innovation diffusion is driven by four factors, namely, innovation, communication channel, time, and social system through which the innovation diffuses (Rogers, 2003). When applied to the context of rumor-mongering in social media, the first two factors are analogous to the rumor message carried on Twitter. Time can be considered as a measurement of the outcome of rumor retransmission while social system refers to the network of users among whom the rumor message finds traction.

\subsection{Theoretical Underpinnings of Rumor Retransmis- sion}

Rumor theory holds that the spread of rumor is associated with at least two elements, namely, anxiety and information ambiguity (Oh, et al., 2013). Anxiety arises because users have a personal stake in the rumor and are hence compelled to act on it. Information ambiguity is an inherent property in the event of a rumor where the truthfulness of what swirls around cannot be quickly established.

Amid the frequent and wild retransmission of information, two theories can be used to explain how users process the rumor message. First, the Elaboration Likelihood Model (ELM) (Cacioppo \& Petty 1984; Zha, et al., 2016) proposes two possible routes. The central route is taken when users possess the cognitive ability and motivation to evaluate the message. The less able and motivated the users, the more the peripheral route will be used. With the peripheral route, users will not examine the information thoroughly but instead rely on general impressions and heuristics to make a judgement call.

Next, opinion leadership literature identifies opinion leaders as those who exert a disproportionate amount of influence over the decision-making of others. For example in the Two-Step Flow Theory, information is postulated to flow from the media to opinion leaders who then pass on their own interpretation in addition to the original content to other people (Katz \& Lazarsfeld, 1955). In this way, opinion leaders weld considerable power in shaping how people perceive and act on the information they share.

\subsection{Proposed Rumor Retransmission Model}

Drawing from the literature on information diffusion, ELM and opinion leadership presented earlier, a rumor retransmission model is proposed. Specifically, it comprises three main constructs, namely, message, user and retransmission outcomes.

Message carries the rumor per se and invariably comprises both cognitive and socio-affective features, reflecting information ambiguity and anxiety as highlighted by rumor theory (Oh, et al., 2010). With only a thin slice of information at hand, users are unable to process it with rigor. Thus, according to ELM, users tend to take the peripheral route and heuristically look for cues of information credibility. In the context of Twitter, the available cognitive cues include the presence of the image, length of the message, the use of URLs and Hashtags.

Image has been recognized as an important cognitive feature in retransmission on Twitter (Liu, et al., 2014). A message which is augmented with an image conveys richer meaning compared to one that is text-only. Moreover, the inclusion of an image tends to bolster the credibility of 
the message (Guan, et al., 2014) because it represents a cue that is difficult to manipulate. In the absence of any objective verification mechanism, an online rumor supported by an image is perceived as believable and hence attracts retransmission. Hence, the first hypothesis is as follows:

Hypothesis 1a: Image in the rumor message affects retransmission outcomes

Message length and the use of URLs have been considered as quality indicators for messages (Chai, et al., 2013). A longer message can carry more content and appears more substantive than a shorter one (Ghose and Ipeirotis, 2011). As a result, the longer message seems more persuasive and leads to a higher probability of retransmission. Furthermore, the use of URLs enables users to draw on external resources on the Internet to convey more content than what Twitter's 140 characters limit would allow (Honeycutt and Herring, 2009). In the case of rumor tweets, lengthy information and the use of URLs signal trustworthiness and inspire confidence. When exposed to such rumor tweets, users may regard them as a credible information source. Their tweetability is thus boosted. Hence, the next two hypotheses are as follows:

Hypothesis 1b: Length of the rumor message affects retransmission outcomes

Hypothesis 1c: The use of URLs in the rumor message affects retransmission outcomes

Hashtags refer to the inclusion of hash symbol (\#) that marks keywords or specific topics in tweets (Cislaru, 2015) to make tweets searchable. In rumour situations where there is a scarcity of information, users will search for whatever little information they can find. Rumor tweets that are discoverable through their hashtags, therefore, have higher chances of being retweeted (Stieglitz and Linh, 2013). Hence, the fourth hypothesis is as follows:

Hypothesis 1d: The use of hashtags in the rumor message affects retransmission outcomes

As with any social media application, Twitter allows for the message to be laden with a mix of social and emotional tones. Particularly during a rumor outbreak, Twitter is often used to deal with the highly anxious situation. Four socio-affective cues available in Twitter include socialization, emotions, sense of belonging and polarity of the message.

Anxiety increases the need for socialization (Pezzo \& Beckstead, 2006). One of the socializing features afforded by Twitter is the use of @ sign.The @ sign can be used in two communicative contexts. When used in dyadic interactions, it serves as a reply to specific users (Honeycutt \& Herring, 2009), even though the message can still be publicly accessed. When used as a broadcast device, the @ sign allows the message to be trans mitted explicitly to all the senders' followers and not just the person mentioned. In any case, messages that make use of such a socializing feature tend to reach a wider audience, and would have a higher chance of being retransmitted. Hence, the fifth hypothesis is proposed:

Hypothesis 1d: The use of socializing feature in the rumor message affects retransmission outcomes

Emotions refer to the expressions of feelings in a message. They have a strong impact on information sharing and rumor retransmission behavior (Oh, et al., 2013). In particular, an emotionally-charged rumor tweet that either expresses fear and anxiety or comfort and encouragement tends to arouse responses (Stieglitz \& Linh, 2013; Zhao, et al., 2013) as users would seek to engage in the process of collective sense-making in the rumor-mongering context. Hence, the following hypothesis is proposed:

Hypothesis 1f: Emotions in the rumor message affects retransmission outcomes

The sense of belonging refers to the feeling of social connection and involvement with the group (Cheung \& Lee, 2012). Individuals identify themselves with the group when they have a strong sense of belonging to the group. Thus, a user who perceives the sense of belonging expressed in a rumor message is more likely to respond by retransmitting it to the group than one who does not (Stieglitz \& Linh 2013; Zhao, et al., 2013). Hence, the next hypothesis is as follows:

Hypothesis 1g: The sense of belonging in the rumor message affects retransmission outcomes

Polarity refers to the tone of the rumor message which could either be positive, negative or neutral. It is often treated as a separate variable from emotions to specify the direction of the sentiment in the message (Hamad, et al., 2017). Due to the negativity bias phenomenon, negative messages were found to be more viral in terms of volume and immediacy than positive and neutral messages (Tsugawa \& Ohsaki, 2015). However, other works related to information diffusion on Twitter found negative content remained small in number and lasted for short periods (Burnap, et al., 2014). The inconclusive findings call for the investigation into how polarity of a rumor message affects retransmission outcomes. Hence, the following hypothesis is proposed:

Hypothesis $1 \mathrm{~h}$ : Polarity of the rumor message affects retransmission outcomes

The second major construct in the proposed rumor retransmission model is the user. In opinion leadership literature, the classical Two-Step Flow Theory (Katz and Lazarsfeld, 1955) identifies influential users as those who add their own interpretation to the source message before passing it on others. But the emergence of social media has democratized opinion leadership such that or 
dinary users are now empowered to influence others so long as they are well-connected to others. This is why connectivity has been commonly used as a proxy for user influence, and is measured in terms of the number of followers in Twitter studies (Kwak, et al., 2010; Suh, et al., 2010). Another aspect of connectivity is the number of friends. This refers to the number of people with whom a user has followed on Twitter. Prior studies have shown that the number of friends is correlated with retweeting decisions (Suh, et al., 2010; Simon, et al., 2015). In a rumor-mongering context, common users would look to those with higher connectivity for information. Hence, the following hypothesis is proposed:

Hypothesis 2a: User's connectivity affects retransmission outcomes

Activity is another user characteristic that could contribute to retransmission (Chen, et al., 2011). Activity refers to the extent of user participation through posting messages. Users who are active on Twitter tend to have their messages read and retweeted by others (Bae, et al., 2014). However, some prior research has found the converse to be true, possibly due to the problem of information overload (Stieglitz \& Linh, 2013). These inconsistencies call for more investigations. The next hypothesis is as follows:

Hypothesis 2b: User's activity affects retransmission outcomes

Previous work noted that user characteristics contribute indirectly to information diffusion on Twitter. In particular, user characteristics were shown to moderate the effect of message characteristics on information diffusion (van de Velde, et al., 2015). Hence, this paper also explores the moderating effect of user characteristics on the relation ships between rumor message characteristics and retransmission.
Hence, the final two hypotheses are as follows:

Hypothesis 3a: User's connectivity moderates the relationship between the rumor message features and retransmission outcomes

Hypothesis 3b: User's activity moderates the relationship between the rumor message features and retransmission outcomes

The retransmission of messages on Twitter is facilitated by its retweet mechanism. Retransmission has mostly been conceptualized as the volume of retweets (Stieglitz \& Linh, 2013; Lee, et al., 2015; van de Velde, et al., 2015) as this is an explicit measure of the interest generated by a rumor message in the network. However, there are relatively few works that considered two other time outcomes, namely, immediacy and lifespan (Zeng, et al., 2016).

Immediacy measures how fast a rumor spreads through a population and is recognized as an indicator for message virality and user's influence (Stieglitz \& Linh, 2013). Researchers found that tweets with URLs and emotional expression are being retweeted more rapidly (Stieglitz \& Linh, 2013). Lifespan represents the length of time during which a rumor attracts attention. It is also an important retransmission outcome because it reflects the duration within which a rumor remains active in the network. It was found that the usage of URLs and positive message polarity are positively associated with the lifespan of a message (Burnap, et al., 2014). Hence, in the research model, all three retransmission outcomes, namely, volume, immediacy and lifespan are included.

Shown in Figure 1, the proposed rumor retransmission model specifies three main constructs, namely rumor message, user, and retransmission. The three research questions and their corresponding hypotheses are depicted as arrows in the figure.

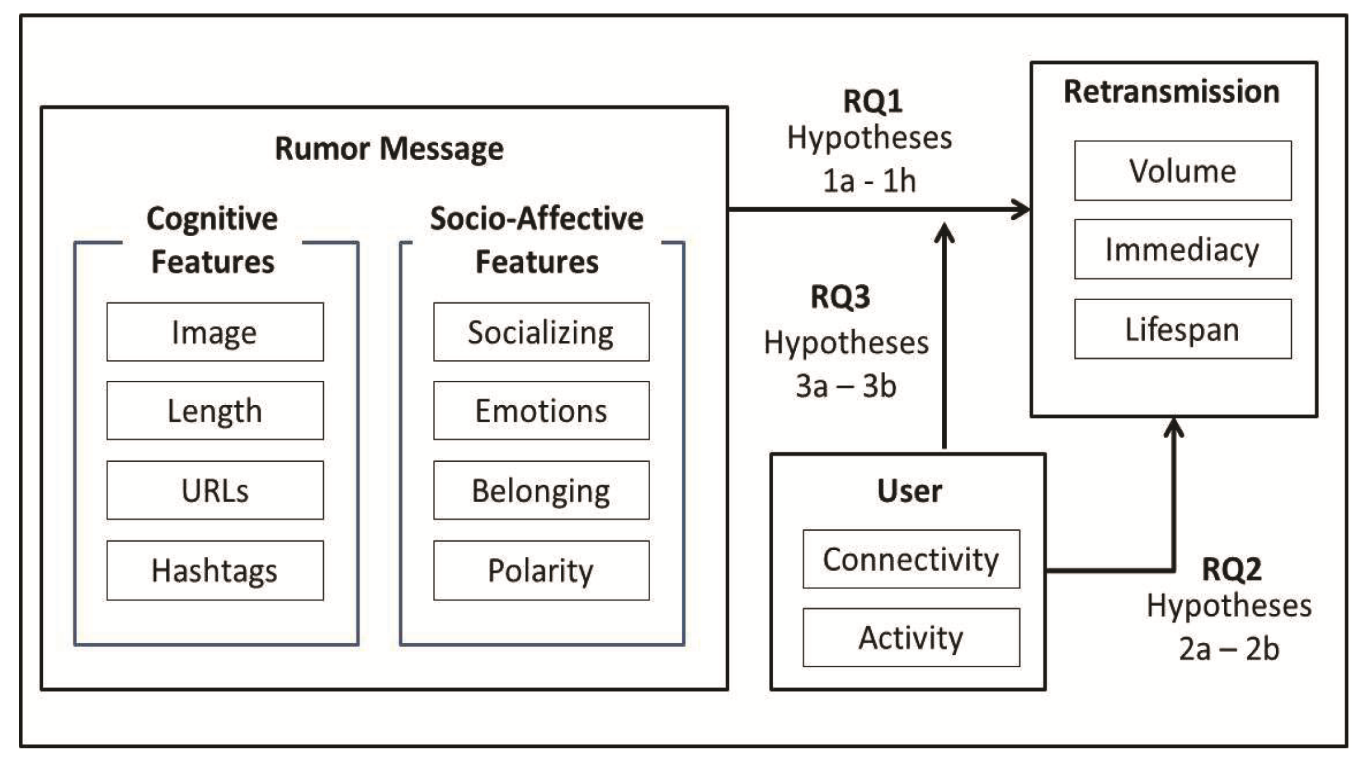

Figure 1. Proposed online rumor retransmission model 
The rapid modernization of Singapore within a single generation has largely been credited to the leadership of founding Prime Minister, Lee Kuan Yew (LKY). With his life interwoven with the history and development of the country, LKY commanded respect from all strata of society. In 2012, the crowd broke into a cheer when they saw him at the annual National Day Parade, dispelling a nagging suspicion at the time that he was either dead or very ill. Apart from personal adoration toward him, there was a sliver of apprehension on whether Singapore could continue to thrive on the global stage in the post-LKY era. Meanwhile, LKY also had his own critics who accused him of being politically ruthless and intolerant of dissent. In any case, his well-being was newsworthy to the Singapore public. When seen in this light, the death hoax of LKY in 2015 presents an interesting context to study a rumor-mongering phenomenon in Singapore.

At 91, LKY was admitted into the hospital in February 2015 for severe pneumonia. When news of his worsening condition was announced on 17 March, a fake screenshot of the Prime Minister's Office's statement flooded the social media the next day. The doctored image that announced LKY's death was circulated at a remarkable speed. It was so widespread that the Prime Minister's Office issued a correction statement and made a police report on the same day. Thereafter, the rumor subsided. Eventually, LKY passed away on 23 March.

\section{Methodology}

\subsection{Data Collection}

The data was collected with the help of a local research center which listens and collects social media data generated by users in near real-time. Prior to this study, data of some 150,000 Singapore Twitter users were already being harvested. The Singapore user set consists of those who publicly share their tweets and identify Singapore as the location in their profile. To establish the user set, a seed set of well-known Singapore users was first selected. Thereafter, their Singapore-based followers and friends were included by snowball sampling repeatedly until the list cannot be expanded further. Tweets from these users were collected using Twitter's Streaming API.

For this purpose of this study, relevant tweets were filtered by \#lky and \#leekuanyew and those created between 18 March 2015, 00:00 hours and 23 March 2015, 03:18 hours. This duration spans the life cycle of LKY's rumored death, starting from the emergence of the fake Prime Minister's Office's screenshot, leading to a spike in the rumor, ending with the Prime Minister's Office's official statement, and eventually his actual demise. Out of 9,309 tweets collected, 460 original tweets related to the rumor which had been retweeted for at least once were identified.

Thereafter, the first retweet and final retweet of each original tweet were found based on their time-stamp. After excluding those with missing retweets, a total of 322 original tweets were admitted as the dataset for analysis. These original tweets had attracted over 5700 retweets which are prefixed with "RT" followed by @username. For each original tweet, the following information was collected: The actual message, time-stamp and user information, including username, location, number of followers and number of friends. Besides, the number of retweet of each original tweet, as well as the time-stamp of its first and final retweet was obtained.

Examples of the actual messages related to the rumored death of LKY include "We all know he's on his way out. No point lying to yourselves, people", "RIP \#LeeKuanYew, Singapore is officially screwed" and "Early reports \#LeeKuanYew has passed away".

\subsection{Operationalization}

Following the research model described above, the independent variables that consist of rumor message and user characteristics were divided into 10 constituents as shown in Table 1.

For rumor message characteristics, the effect of cognitive features, namely Image, Length, URLs, and Hashtags, as well as socio-affective features, namely Socializing, Emotions, Belonging and Polarity were measured. Length is derived from the number of characters of a message. Hashtags refer to the number of hashtags included in the message. Polarity refers to neutral, negative and positive sentiment. They were coded as 0,1 and 2 respectively. Other variables are dichotomous ( 1 for presence; 0 for absence). Image and URL refer to the presence of image and external link respectively (Liu, et al., 2014). Socializing refers to the presence of directed message to a specific user account (@) (Oh, et al., 2013). Emotions are represented by Twitter message that expresses positive and negative emotions. Rumor tweets that contained positive emotional words such as "awesome" and "grateful" and negative emotional words such as "sad" and "grief" will be coded as "1". Belonging emphasizes the sense of togetherness and solidarity with the use of words such as "we", "our" and "together".

User characteristics have two constituents: Connectivity and Activity. Connectivity is operationalized as the ratio of the number of followers to that of friends (Bae, et al., 2014). In Twitter, a follower is one who follows the user while a friend is one whom the user follows. The use of such ratio helps reduce the likelihood of mistaking a spammer for someone who is highly connected. Activity is measured by the ratio of status to age (Bae, et al., 2014). Status refers to the number of tweets posted by the user while age is the number of days since the user's Twitter account was created. Using such a ratio instead of status or age alone offers a better indication of a user's level of activity on Twitter.

Retransmission outcomes comprise three dependent variables, namely, Volume, Immediacy and Lifespan. Volume is the number of retweets, an original tweet attracts. 


\begin{tabular}{|l|l|}
\hline \multicolumn{1}{|c|}{ Variables } & \multicolumn{1}{c|}{ Description } \\
\hline Rumor Message & A tweet with image. \\
\hline Cognitive - Image & Number of characters in a tweet. \\
\hline Cognitive - Length & A tweet with URL. \\
\hline Cognitive - URLs & Number of hashtags in a tweet. \\
\hline Cognitive - Hashtags & A tweet that directs to specific user account (@). \\
\hline Socio-Affective - Socializing & A tweet that expresses emotions. \\
\hline Socio-Affective- Emotions & A tweet that emphasizes on sense of belonging. \\
\hline Socio-Affective- Belonging & The neutral, negative or positive sentiment of a tweet. \\
\hline Socio-Affective - Polarity & \multicolumn{2}{|l|}{} \\
\hline User & Ratio of user's number of followers to number of friends. \\
\hline Connectivity & Ratio of number of tweets a user posted to number of days he created Twitter account. \\
\hline Activity & \multicolumn{2}{|c|}{ The number of retweets an original tweet attracts. } \\
\hline Retransmission & The time lag between an original tweet and its first retweet. \\
\hline Volume & The time difference between an original tweet and its final retweet. \\
\hline Immediacy & \\
\hline Lifespan & \\
\hline
\end{tabular}

Table 1. Characteristics contributing to retransmission of rumor tweets

Immediacy is the time lag between an original tweet and its first retweet. Lifespan is the time period between an original tweet and its final retweet. Both Immediacy and Lifespan were calculated in seconds (Burnap, 2014).

Except for Emotions, Belonging, and Polarity, all the variables were coded objectively by extracting directly from the dataset. For Emotions and Belonging which required qualitative judgement from human coders, a pilot set of 100 tweets was assigned independently to two coders. The Cohen's Kappa of Emotions and Belonging were found to be 0.87 and 0.8 respectively, indicating non-chanced agreement. The remaining set was divided equally among them for further coding.

For Polarity, sentiment analysis program SentiStrength was used to measure the strength of positive and negative sentiment in Twitter messages (Stieglitz \& Linh, 2013). A message was coded as 0 (neutral) when the resulting score is 0 . It was coded as 1 (negative) when the resulting score is -1 or lower, and coded as 2 (positive) when the resulting score is 1 or higher. After coding, two dummy variables were created for negative (Negative Tone) and positive Polarity (Positive Tone).

\subsection{Data Analysis}

To study how rumor message and user characteristics affect retransmission of tweets in terms of Volume (part of RQ1 and RQ2), negative binomial regression was used so as to allow for over-dispersion in the dataset. To study how rumor message and user characteristics affect retransmission of tweets in terms of Immediacy and Lifespan (part of RQ1 and RQ2), ordinary least squares (OLS) regression and Cox regression was used respectively (Stieglitz \& Linh, 2013; Burnap, et al., 2014). To account for non-normality, Immediacy and Lifespan were log-transformed before the analysis (Stieglitz \& Linh, 2013).

To address RQ3, interaction terms were created. Independent variables were first mean-centered, and then multiplied by the rumor message characteristics (predictor) and user characteristics (moderator) to reduce multicollinearity problem. In this paper, rumor message characteristics comprise nine items (including two dummy variables of Polarity), and user characteristics comprise two items. Hence, the interaction terms represent 18 items $(9 \times 2)$. The statistical analyses were done using SPSS 22.0 software.

\section{Results}

The descriptive statistics for the data set are summarized in Table 2.

Statistical results of the research model are summarized in Table 3, and their interpretations are presented in Section 6 later. Due to space constraints, only interaction terms with significant effects are shown. $R^{2}$ for negative 


\begin{tabular}{|l|c|c|c|}
\hline Variables & Range & Mean & SD \\
\hline Rumor Message & & & \\
\hline Image & $0-1$ & 0.3 & 0.46 \\
\hline Length & $7-146$ & 98.27 & 36.89 \\
\hline URLs & $0-1$ & 0.11 & 0.31 \\
\hline Hashtags & $1-4$ & 1.44 & 0.66 \\
\hline Socializing & $0-1$ & 0.31 & 0.47 \\
\hline Emotions & $0-1$ & 0.27 & 0.5 \\
\hline Belonging & $0-1$ & 0.21 & 0.41 \\
\hline Polarity & $0-2$ & 0.88 & 0.79 \\
\hline User & & & \\
\hline Connectivity & $0-4934.37$ & 35.57 & 298.23 \\
\hline Activity & $0-119.49$ & 15 & 18.81 \\
\hline Retransmission & & & \\
\hline Volume & $1-1370$ & 17.82 & 90.29 \\
\hline $\log$ (Immediacy) & $0.78-5.19$ & 2.30 & 0.78 \\
\hline $\log$ (Lifespan) & $0.90-5.55$ & 3.19 & 1.05 \\
\hline
\end{tabular}

Table 2. Descriptive statistics $(N=322)$

\begin{tabular}{|c|c|c|c|c|c|c|c|c|c|c|}
\hline & \multicolumn{2}{|c|}{ Model 1} & \multicolumn{2}{|c|}{ Model 2} & \multirow{2}{*}{\multicolumn{2}{|c|}{\begin{tabular}{|l|r|} 
Model 3 & Model 4 \\
\multicolumn{2}{|c|}{ Effect on Immediacy }
\end{tabular}}} & \multicolumn{2}{|c|}{ Model 5} & \multicolumn{2}{|c|}{ Model 6} \\
\hline & \multicolumn{4}{|c|}{ Effect on Volume } & & & \multicolumn{4}{|c|}{ Effect on Lifespan } \\
\hline & $\beta(\mathrm{SE})$ & $\operatorname{Exp}(\beta)$ & $\beta$ (SE) & $\operatorname{Exp}(\beta)$ & $\beta(\mathrm{SE})$ & $\beta$ (SE) & $\beta$ (SE) & $\operatorname{Exp}(\beta)$ & $\beta(\mathrm{SE})$ & $\operatorname{Exp}(\beta)$ \\
\hline Image & $\begin{array}{c}1.968^{* * *} \\
(0.159) \\
\end{array}$ & 7.158 & $\begin{array}{l}2.02 * * * \\
(0.198) \\
\end{array}$ & 7.536 & $\begin{array}{c}0.208 * * \\
(0.1)\end{array}$ & $\begin{array}{c}0.296 * * \\
(0.12)\end{array}$ & $\begin{array}{c}0.499 * * * \\
(0.146) \\
\end{array}$ & 0.607 & $\begin{array}{l}0.64 * * * \\
(0.176)\end{array}$ & 0.527 \\
\hline Length & $\begin{array}{c}0.009 * * * \\
(0.002)\end{array}$ & 1.009 & $\begin{array}{l}0.011 * * \\
(0.005)\end{array}$ & 1.011 & $\begin{array}{l}-0.001 \\
(0.001) \\
\end{array}$ & $\begin{array}{l}-0.004 \\
(0.003) \\
\end{array}$ & $\begin{array}{l}-0.003 \\
(0.002) \\
\end{array}$ & 0.997 & $\begin{array}{l}-0.005 \\
(0.005)\end{array}$ & 0.995 \\
\hline URLs & $\begin{array}{c}-0.914 * * * \\
(0.237)\end{array}$ & 0.401 & $\begin{array}{c}-1.029 * * * \\
(0.278)\end{array}$ & 0.357 & $\begin{array}{l}0.284 * * \\
(0.139)\end{array}$ & $\begin{array}{l}0.408 * * \\
(0.161)\end{array}$ & $\begin{array}{l}-0.191 \\
(0.193)\end{array}$ & 0.826 & $\begin{array}{l}-0.008 \\
(0.233)\end{array}$ & 0.992 \\
\hline Hashtags & $\begin{array}{c}-0.374 * * * \\
(0.114)\end{array}$ & 0.688 & $\begin{array}{l}-0.212 \\
(0.169)\end{array}$ & 0.809 & $\begin{array}{l}0.157^{* * *} \\
(0.066)\end{array}$ & $\begin{array}{c}0.086 \\
(0.099)\end{array}$ & $\begin{array}{c}0.080 \\
(0.094)\end{array}$ & 1.083 & $\begin{array}{l}-0.016 \\
(0.149)\end{array}$ & 0.984 \\
\hline Socializing & $\begin{array}{c}-1.288^{* * * *} \\
(0.154)\end{array}$ & 0.276 & $\begin{array}{c}-1.288 * * * \\
(0.198)\end{array}$ & 0.276 & $\begin{array}{c}0.266^{* * * *} \\
(0.1)\end{array}$ & $\begin{array}{l}0.209 * \\
(0.122)\end{array}$ & $\begin{array}{c}0.225 \\
(0.139)\end{array}$ & 1.252 & $\begin{array}{c}0.33 * \\
(0.181)\end{array}$ & 1.391 \\
\hline Emotions & $\begin{array}{l}0.251^{*} \\
(0.154)\end{array}$ & 1.286 & $\begin{array}{c}0.265 \\
(0.176) \\
\end{array}$ & 1.303 & $\begin{array}{c}0.13 \\
(0.091) \\
\end{array}$ & $\begin{array}{c}0.057 \\
(0.101)\end{array}$ & $\begin{array}{c}0.143 \\
(0.134)\end{array}$ & 1.154 & $\begin{array}{c}0.124 \\
(0.155)\end{array}$ & 1.132 \\
\hline Belonging & $\begin{array}{c}0.461 * * * \\
(0.183) \\
\end{array}$ & 1.586 & $\begin{array}{c}0.565 \\
(0.689) \\
\end{array}$ & 1.76 & $\begin{array}{c}0 \\
(0.110) \\
\end{array}$ & $\begin{array}{c}0.204 \\
(0.418) \\
\end{array}$ & $\begin{array}{l}-0.249 \\
(0.158) \\
\end{array}$ & 0.779 & $\begin{array}{l}-0.697 \\
(0.561) \\
\end{array}$ & 0.498 \\
\hline Positive Tone & $\begin{array}{c}-0.563 * * * \\
(0.162)\end{array}$ & 0.57 & $\begin{array}{c}-1.625 * * * \\
(0.522)\end{array}$ & 0.197 & $\begin{array}{c}0.172 \\
(0.110)\end{array}$ & $\begin{array}{c}0.435 \\
(0.278)\end{array}$ & $\begin{array}{c}0.130 \\
(0.154)\end{array}$ & 1.139 & $\begin{array}{c}0.136 \\
(0.385)\end{array}$ & 1.146 \\
\hline $\begin{array}{l}\text { Negative } \\
\text { Tone }\end{array}$ & $\begin{array}{c}-0.458^{* * * *} \\
(0.15)\end{array}$ & 0.633 & $\begin{array}{l}-0.367 * \\
(0.196)\end{array}$ & 0.693 & $\begin{array}{c}0.096 \\
(0.099)\end{array}$ & $\begin{array}{c}0.067 \\
(0.124)\end{array}$ & $\begin{array}{c}0.128 \\
(0.137)\end{array}$ & 1.137 & $\begin{array}{l}-0.002 \\
(0.183)\end{array}$ & 0.998 \\
\hline Connectivity & $\begin{array}{c}0.001 * * * \\
(0.0002)\end{array}$ & 1.001 & $\begin{array}{l}-0.004 \\
(0.006)\end{array}$ & 0.996 & $\begin{array}{c}0 \\
(0)\end{array}$ & $\begin{array}{c}0.002 \\
(0.003)\end{array}$ & $\begin{array}{c}0 \\
(0)\end{array}$ & 1.000 & $\begin{array}{c}0.004 \\
(0.005)\end{array}$ & 1.004 \\
\hline Activity & $\begin{array}{l}-0.002 \\
(0.004)\end{array}$ & 0.998 & $\begin{array}{c}0 \\
(0.014)\end{array}$ & 1.000 & $\begin{array}{l}0.006 * * \\
(0.002)\end{array}$ & $\begin{array}{c}0.002 \\
(0.008)\end{array}$ & $\begin{array}{c}0.002 \\
(0.003)\end{array}$ & 1.002 & $\begin{array}{l}-0.003 \\
(0.012)\end{array}$ & 0.997 \\
\hline $\begin{array}{l}\text { Connectivity } \\
\text { x Image }\end{array}$ & & & & & & $\begin{array}{l}0.006 * * \\
(0.003)\end{array}$ & & & & \\
\hline $\begin{array}{l}\text { Connectivity } \mathrm{x} \\
\text { Positive Tone }\end{array}$ & & & $\begin{array}{c}-0.034 * * \\
(0.015)\end{array}$ & 0.967 & & & & & & \\
\hline $\begin{array}{l}\text { Activity } x \\
\text { Image }\end{array}$ & & & & & & $\begin{array}{c}0.019 * * * \\
(0.007)\end{array}$ & & & $\begin{array}{l}-0.019 * \\
(0.012)\end{array}$ & 0.981 \\
\hline $\begin{array}{l}\text { Activity } \mathrm{x} \\
\text { Socializing }\end{array}$ & & & & & & $\begin{array}{c}0.021 * * * \\
(0.008)\end{array}$ & & & & \\
\hline $\begin{array}{l}\text { Activity } \mathrm{x} \\
\text { Belonging }\end{array}$ & & & & & & & & & $\begin{array}{l}0.024 * * \\
(0.011)\end{array}$ & 0.977 \\
\hline $\begin{array}{l}\text { Activity } \mathrm{x} \\
\text { Positive Tone }\end{array}$ & & & $\begin{array}{c}0.034 * * * \\
(0.012)\end{array}$ & 1.035 & & $\begin{array}{l}-0.014 * \\
(0.008)\end{array}$ & & & & \\
\hline $\mathrm{R}^{2}$ & 0.17 & & 0.19 & & 0.11 & 0.18 & 0.064 & & 0.14 & \\
\hline
\end{tabular}

Note: $\beta=$ estimated coefficient; $\mathrm{SE}=$ standard errors; $\operatorname{Exp}(\beta)=$ exponentiated estimated coefficient; ${ }^{*} p<.1 ; * * p<.05 ; * * * p<.01$.

Table 3. Regression results $(N=322)$ 
binomial regression is reported by McFadden's pseudo $R^{2}$, while the $R^{2}$ of Cox regression is reported by generalized $R^{2}$. Prior to the analysis, the values of variance inflation factor (VIF) were inspected to check for potential multicollinearity. The highest VIF value was less than 3 , confirming that multicollinearity was not a problem.

Model 1 and 2 show the results for negative binomial regression. Model 1 presents the results of the direct effect of rumor message and user characteristics on Volume $\left(R^{2}=0.17\right)$, while Model 2 shows the results of the overall model which includes direct and indirect effects on Volume $\left(R^{2}=0.19\right)$. The omnibus test showed that the models are statistically significant. Model 3 and 4 present the results for OLS regression. They show the results of direct effect $\left(R^{2}=0.11\right)$ and overall model $\left(R^{2}=0.18\right)$ on Immediacy respectively. In Model 3 and 4 , a positive estimate is interpreted as longer time lag, and therefore suggests reduced retransmission immediacy. The results for Cox regression are shown in Model 5 and 6 . They present the results of direct effect $\left(R^{2}=0.064\right)$ and overall model $\left(R^{2}=\right.$ 0.14 ) on Lifespan respectively. The diagnostics for the full model indicated a good fit to the data $(-2$ Log-likelihood $=$ $2883.521, p<.001)$. In Model 5 and 6 , a positive estimate is interpreted as increasing hazards to survival, and hence points to a shortened lifespan of the messages.

All rumor message characteristics were found to have significant relationships with Volume (Model 1$)$. In particular, Image $(\operatorname{Exp}(\beta)=7.158)$, Length $(\operatorname{Exp}(\beta)=1.009)$, Emotions $(\operatorname{Exp}(\beta)=1.286)$, and Belonging $(\operatorname{Exp}(\beta)=$ $1.586)$ are positively related, while URLs $(\operatorname{Exp}(\beta)=0.401)$, Hashtags $(\operatorname{Exp}(\beta)=0.688)$, Socializing $(\operatorname{Exp}(\beta)=0.276)$, positive Polarity $(\operatorname{Exp}(\beta)=0.57)$, and negative Polarity $(\operatorname{Exp}(\beta)=0.633)$ are negatively related. Moreover, Image $(\beta=-0.208)$ is positively related to Immediacy whereas URLs $(\beta=0.284)$, Hashtags $(\beta=0.157)$ and Socializing $(\beta=0.266)$ are negatively related (Model 3$)$. As for the relationships with Lifespan, Image $(\operatorname{Exp}(\beta)=0.527)$ is positively related while Socializing $(\operatorname{Exp}(\beta)=1.391)$ is negatively related (Model 6).

In other words, Hypotheses $1 \mathrm{a}$ and $1 \mathrm{e}$ are supported across all three retransmission outcomes. Hypotheses $1 \mathrm{c}$ and $1 \mathrm{~d}$ are supported in terms of Volume and Immediacy as retransmission outcomes. Hypotheses $1 \mathrm{~b}, 1 \mathrm{f}$, $1 \mathrm{~g}$ and $1 \mathrm{~h}$ are supported only in terms of Immediacy as retransmission outcome.

For user characteristics, Connectivity $(\operatorname{Exp}(\beta)=1.001)$ is positively correlated to Volume (Model 1 ), while Activity $(\beta=-0.006)$ is positively associated with Immediacy (Model 3 ). However, none of the user characteristics was found to have any significant relationship with Lifespan. In other words, Hypotheses $2 \mathrm{a}$ and $2 \mathrm{~b}$ are supported only in terms of Volume and Immediacy as retransmission outcomes respectively.

Regarding the moderating effect, Connectivity $(\operatorname{Exp}(\beta)=$
0.967) and Activity $(\operatorname{Exp}(\beta)=1.035)$ moderated relationship between positive Polarity and Volume (Model 2). Besides, both Connectivity $(\beta=-0.006)$ and Activity $(\beta=$ $0.019)$ moderated the relationship between Image and Immediacy (Model 4). Activity positively moderated the effect of Socializing $(\beta=-0.021)$ and positive Polarity $(\beta$ $=-0.014$ ) on Immediacy (Model 4). Activity also moderated the effect of Image $(\operatorname{Exp}(\beta)=0.981)$ and Belonging $(\operatorname{Exp}(\beta)=0.977)$ on Lifespan (Model 6).

In other words, Hypothesis $3 a$ is supported under the following cases, namely, Polarity as message characteristic and Volume as retransmission outcome; and Image as message characteristic and Immediacy as retransmission outcome. Hypothesis $3 \mathrm{~b}$ is supported under the following cases, namely, Polarity as message characteristic and Volume as retransmission outcome; Image, Socializing and Polarity as message characteristics and Immediacy as retransmission outcome; and Image and Belonging as message characteristics and Lifespan as retransmission outcome.

\section{Discussion}

Arising from the statistical analysis, four major findings can be drawn. First, the results confirm the robustness of the research model (Model 1 to 6 ) and show that the concept of information diffusion can be applied to online rumor retransmission studies (Stieglitz \& Linh, 2013; Suh, et al., 2010; Burnap, et al., 2014). Findings show that rumor message and user characteristics of tweets have significant effects on retransmission volume $\left(R^{2}=0.19, p<\right.$ $.001)$, immediacy $\left(R^{2}=0.18, p<.001\right)$, and lifespan $\left(R^{2}=0.14\right.$, $p<.001)$. In particular, all rumor message and user characteristics except Activity were found to be significant for Volume (Model 1). Furthermore, Image, URLs, Hashtags, Socializing, and Activity are statistically significant for Immediacy (Model 3), whereas Image and Socializing are significant for Lifespan (Model 6). As postulated by ELM, users look for peripheral cues of credibility afforded by Twitter when they are unable to process information rigorously. Hence, messages that exploit such cues have a higher chance of being retweeted.

Second, Image and Socializing are significant features across the three retransmission outcomes. The former is positively associated while the latter is negatively associated with Volume, Immediacy and Lifespan (Model 1, 3 and 5). Consistent with prior literature (Liu, et al., 2014; Guan, et al., 2014), a rumor message in the text which is supplemented by an image becomes more visually captivating. According to media richness theory (Lengel \& Daft, 1989), the capability of a medium to handle multiple information cues simultaneously is an important characteristic of its richness. In this case, tweets that are informationally enriched with images make for more effective communication. In fact, most of the images which circulated widely were photos of LKY, the doctored screenshot of the Prime Minister's Office's announcement, and photos of media waiting at the hospital where he was 
staying. Users were apparently taken in by rumor messages having these photos. As a well-respected political figure in Singapore, information about his health was of national interest. Tweets that carried his photos and health information thus attracted retransmission not just in terms of volume but the speed and also the long duration within they remain active in the network. The strong negative correlation between Socializing and retransmission meant that tweets intended either for dyadic interactions or to be broadcast to a group of followers do not end up being retweeted. They are, after all, used to forge social ties rather than achieve virality.

Third, contrary to prior research (Stieglitz \& Linh, 2013; Suh, et al., 2010; van de Velde, et al., 2015), the findings indicate that the use of URLs and Hashtags resulted in less retweets and lower immediacy (Model 1 and 3). A possible explanation might be that in the case of inauspicious contexts such as the rumored death of a political figure, straightforward, simple rumor messages tend to resonate with users (Lee, et al., 2015). Any additional effort to click the URLs to obtain more information or deliberate search using hashtags represents a barrier to retransmission (Zeng et al., 2016). Another reason could be that tweets having URLs and hashtags could be topically different from those without URL or hashtag. With low interests and perceived personal involvement in these tweets, their retransmission is likely to be curtailed.

Fourth, user characteristics were found to have both direct and indirect effects on rumor retransmission. As postulated by opinion leadership literature, influential users are able to shape the perception of other users, resulting in their messages being retweeted. With a more granular treatment to the notion of opinion leadership, it is interesting to note that retransmission outcomes between those with high connectivity and those with high activity differ. Specifically, highly connected users are able to generate more retweets. Perhaps by virtue of their wide social network, there is a greater chance for their messages to be echoed. On the other hand, users who are highly active are able to generate retweets more quickly. It seems that having been accustomed to their high frequency of Twitter use, followers of these influential users are also frequent users of Twitter who respond promptly by retweeting.

In terms of the nature of the relationship between socioaffective features and retransmission, prior literature has not been consistent (Tsugawa \& Ohsaki, 2015; Burnap, et al., 2014). To augment these studies, it was found that user characteristics moderate the relationship between socio-affective features and the three retransmission outcomes. In addition, the findings show that positive sentiment rumor messages posted by active users are positively related to Volume and Immediacy. Probably because LKY was fondly recognized as Singapore's founding father, the rumor content about his death might rouse users' gratitude and nationalistic identity, which in turn, fuelled retweets.

\section{Conclusion}

This paper examined the rumor message and user characteristics that could affect the retransmission of rumor tweets in terms of volume, immediacy and lifespan. In addressing RQ1, with respect to Volume, the following variables, namely Image, Length, Emotions, and Belonging are positively related, while URLs, Hashtags, Socializing, and Polarity are negatively related. With respect to Immediacy, Image is positively related, while URLs, Hashtags and Socializing are negatively related. With respect to Lifespan, Image is positively related while Socializing is negatively related.

In addressing RQ2, Connectivity and Activity are positively related to Volume and Immediacy respectively. There is no significant relationship between user characteristics and Lifespan. In addressing RQ3, user characteristics have a moderating effect on the relationships between rumor message characteristics and retransmission outcomes. Both Connectivity and Activity moderate the relationship between positive Polarity and Volume. Connectivity moderates the effect of Image on Immediacy, while Activity moderates the effect of Image, Socializing and positive Polarity on Immediacy. Moreover, Activity also moderates the effect of Image and Belonging on Lifespan.

This paper offers a number of implications for both theory and practice. On the theoretical front, it enriches the ongoing research by illustrating the applicability of the information diffusion model on online rumor retransmission which hitherto has yet to be attempted. User characteristics were shown to exert a direct as well as moderating effect on retransmission outcomes. Moreover, this paper demonstrates how online rumor retransmission outcomes can be granularized into volume, immediacy, and lifespan, all of which are related but distinct constructs that were neglected in previous studies.

On the practical front, this paper has implications for organizations including government agencies and companies seeking to online combat rumors. First, organizations should pay more attention to any lengthy messages that carry images because they tend to attract retransmission. Second, due to the influence of user characteristics on retransmission, organizations should regularly monitor messages posted by established users of Twitter. Finally, organizations need to craft rumor-correction messages in such a way that would spread widely in a short time and for a longer period. As this paper suggests, messages that are brief, clear, without the use of “@” signs, URLs, hashtags would be ideal. Besides, the addition of photo and expressions of positive emotions in rumor-correction messages should also be considered.

Three limitations in this paper must be acknowledged. First, the analysis was based on one case study about a political figure, drawing data from a single social media platform. Due to the sparse social network among users in the dataset, there was no opportunity to study rumor 
retransmission patterns based on users' perception of the variety of source nodes. One of the possible future research directions would be to apply the research model to the retransmission of rumor-debunking messages. Next, the proposed model does not control for the themes of the tweets. A thematic analysis could have provided additional clues as to why some tweets attracted more retransmission than others. In the case of the LKY's death hoax, most rumor tweets were about his purported demise without much content variation. But for future research examining rumor cases with different sub-themes, it will be worthwhile to incorporate tweet topics as a construct. Finally, retransmission of tweets is analogous to the information selection in other contexts, such as newspaper and TV programs. It is also significant to consider other factors that may affect the retransmission, such as the receivers' initial belief, and the timeliness and relatedness of the information exposure to them. Moreover, without having direct access to users on Twitter, it was impossible to determine the actual motivation for retransmitting the rumor tweets. Conducting user study involving interviews is likely to enhance the results' accuracy.

\section{References}

[1] Bae, Y., Ryu, P., Kim, H. (2014), Predicting the lifespan and retweet times of tweets based on multiple feature analysis, ETRI Journal, 3, 418-428.

[2] Bailey, N. (1975), The mathematical theory of infectious diseases and its applications, 2nd edition. Griffin, London. 1975.

[3] Burnap, P., Williams, M., Sloan, L., Rana, O., Housley, W., Edwards, A., Knight, V. Procter, Voss, A. (2014), Tweeting the terror: modelling the social media reaction to the Woolwich terrorist attack, Social Network Analysis and Mining, 4(1) 1-14.

[4] Cacioppo, J. T., Petty, R. E. (1984). The Elaboration Likelihood Model of persuasion, Advances in Consumer Research, 11, 673-675.

[5] Chai, W., Xu, W., Zuo, M., Wen, X. (2013). ACQR: A novel framework to identify and predict influential users in micro-blogging, In: Proceedings of the Pacific Asia Conference on Information Systems (PACIS), AISel. Article 20.

[6] Chen, G. (2011). Tweet this: A uses and gratifications perspective on how active Twitter use gratifies a need to connect with others. Computers in Human Behavior, 27 (2) 755-762.

[7] Cheung, C., Lee, M. (2012). What drives consumers to spread electronic word of mouth in online consumeropinion platforms, Decision Support Systems, 53(1) 218225.

[8] Chua, A., Tee, C-Y., Pang, A., Lim, E-P. (2016). The Retransmission of rumor-related tweets: Characteristics of source and message. Proceedings of Society Media and Society, July 11-13, London, UK, Article No. 22, New York: ACM
[9] Chua, A., Tee, C-Y., Pang, A., Lim, E.-P. (2017). The retransmission of rumor and rumor correction messages on Twitter, American Behavioral Scientist, 61 (7) 707-723.

[10] Cislaru, G. (2015). Emotions in tweets: From instantaneity to preconstruction, Social Science Information, 54 (4) 455-469.

[11] DiFonzo, N., Bordia, P. (2017). Rumor psychology: Social and organizational approaches. Washington DC: American Psychological Association.

[12] Ghose, A., Ipeirotis, P. G. (2011). Estimating the Helpfulness and Economic Impact of Product Reviews: Mining Text and Reviewer Characteristics, IEEE Transactions on Knowledge and Data Engineering, 23 (10) 1498-1512.

[13] Guan, W., Gao, H., Yang, M., Li, Y., Ma, H., Qian, W., Yang, X. (2014). Analyzing user behavior of the micro-blogging website Sina Weibo during hot social events, Physica A: Statistical Mechanics and its Applications, 395, 340-351.

[14] Hamad, R. A., Alqahtani, S. M., Torres, M. T. (2017). Emotion and polarity prediction from Twitter, In: Proceedings of Computing Conference, IEEE, 297-306.

[15] Honeycutt, C., Herring, S. C. (2009). Beyond microblogging: Conversation and collaboration via Twitter, In: Proceedings of the 42nd Hawaii International Conference on System Sciences (HICSS-42), IEEE, 1-10.

[16] Katz, E., Lazarsfeld, P. F. (1955). Personal Influence, the Part Played by People in the Flow of Mass Communications. NY: Transaction Publishers.

[17] Kimmel. (2013). A Rumors and rumor control: A manager's guide to understanding and combatting rumors. NY: Routledge.

[18] Kwak, H., Lee, C., Park, H., Moon, S. (2010). What is Twitter, A social network or a news media?, Proceedings of the 19th International Conference on World Wide Web, ACM Digital Library, 591-600.

[19] Lee, J., Agrawal, M., Rao, H. (2015). Message diffusion through social network service: The case of rumor and non-rumor related tweets during Boston bombing 2013, Information Systems Frontiers, 17 (5) 997-1005.

[20] Lengel, R., Daft, R. L. The selection of communication media as an executive skill. The Academy of Management Executive 1989. 2 (3) 225-232.

[21] Liu, F., Burton-Jones, A., Xu, D. (2014). Rumors on social media in disasters: Extending transmission to retransmission, In: Proceedings of the Pacific Asia Conference on Information Systems (PACIS), Article 49, AISel.

[22] Ma, J., Luo, Y. (2019). The classification of rumour standpoints in online social network based on combinatorial classifiers, Journal of Information Science Epub ahead of print 21 Feb 2019. DOI: 10.1177/0165551519828619

[23] Maddock, J., Starbird, K., Al-Hassani, H. J. , Sandoval, D. E. , Orand, M., Mason, R. M. (2015). Characterizing online rumoring behavior using multi-dimensional signatures, 
In: Proceedings of the ACM Conference on Computer Supported Cooperative Work \& Social Computing, ACM, 228-241.

[24] Marett, K., Joshi, K. (2009). The decision to share information and rumors: Examining the role of motivation in an online discussion forum, Communications of the Association for Information Systems, 24(1) 47-68.

[25] Morris. S. (2000). Contagion, Review of Economic Studies, 67, 57-78.

[26] Mozafari, N., Hamzeh, A. (2015). An enriched social behavioural information diffusion model in social networks, Journal of Information Science, 41(3) 273-283.

[27] Oh, O., Agrawal, M., Rao, H. (2013). Community intelligence and social media services: A rumor theoretic analysis of tweets during social crises, MIS Quarterly 37 (2) 407-426.

[28] Oh, O., Kwon, K. H., Rao, H. (2010). An exploration of social media in extreme events: rumor theory and twitter during the Haiti Earthquake 2010. In: Proceedings of the 31st International Conference on Information Systems. Paper 231.

[29] Pal, A., Chua, A., Goh, D. H. (2017). Does KFC sell rat? Analysis of tweets in the wake of a rumor outbreak, Aslib Journal of Information Management, 69 (6) 660-673.

[30] Pezzo, M. V., Beckstead, J. W. (2006). A Multilevel Analysis of Rumor Transmission: Effects of Anxiety and Belief in Two Field Experiments, Basic and Applied Social Psychology, 28 (1) 91-100.

[31] Rogers, E. M. (2003). Diffusion of Innovations, 5th ed., NY: The Free Press.

[32] Simon, T., Goldberg, A., Adini, B. (2015). Socializing in emergencies-A review of the use of social media in emergency situations, International Journal of Information Management, 35 (5) 609-619.

[33] Statista. (2019). Leading countries based on number of Twitter users as of January 2019 (in millions), https:// www.statista.com/statistics/242606/number-of-active-twitter-users-in-selected-countries/. (accessed 12 April 2019)

[34] Stieglitz, S., Linh, D-X. (2013). Emotions and information diffusion in social media-sentiment of microblogs and sharing behavior, Journal of Management Information Systems, 29 (4) 217-248.

[35] Suh, B., Hong, L., Pirolli, P., Chi, E. (2010). Want to be Retweeted? Large Scale Analytics on Factors Impacting Retweet in Twitter Network, In: Proceedings of the 2nd International Conference on Social Computing, IEEE Xplore Digital Library, 177-184.

[36] Tsugawa, S., Ohsaki, H. (2015). Negative messages spread rapidly and widely on social media, In: Proceedings of the Conference on Online Social Networks (COSN), ACM Digital Library, 151-160.

[37] Van de Velde, B., Meijer, A., Homburg, V. (2015). Police message diffusion on Twitter: analysing the reach of social media communications, Behaviour \& Information Technology, 34(1) 4-16.

[38] Zeng, L., Starbird, K., Spiro, E. (2016). Rumors at the speed of light? Modeling the rate of rumor transmission during crisis, In: Proceedings of the 49th Hawaii International Conference on System Sciences (HICSS), IEEE, 1969-1978.

[39] Zha, X., Li, L., Yan, Y., Wang, Q., Wang, G. (2016). Exploring digital library usage for getting information from the ELM perspective: the moderating effect of information need, Aslib Journal of Information Management, 68 (3) 286-305.

[40] Zhang, Y., Chen, C. (2014). A rumor spreading model considering latent state, In: Proceedings of the International Conference on Management Science and Engineering Management, Springer Berlin Heidelberg, 155-162.

[41] Zhao, D., Wang, F., Wei, J., Liang, L. (2013). Public reaction to information release for crisis discourse by organization: Integration of online comments, International Journal of Information Management, 33(3) 485-495. 